\title{
Comparing strategies to estimate the association of obesity with mortality via a Markov model
}

\author{
Bingshu E. Chen* ${ }^{* \dagger}$, Barry I. Graubard ${ }^{\ddagger}$, \\ Katherine M. FlegaL ${ }^{\S}$ AND Mitchell H. GAiL ${ }^{\ddagger}$
}

We used a first order discrete Markov model to investigate strategies to obtain unbiased estimates of the relative mortality hazard for comparing obese with non-obese participants. This hazard ratio is confounded by the fact that obese participants can be either sick or well, as can non-obese participants, and participants can migrate over time from their initial classification on obesity and health status. The parameters of the model were estimated from national survey data and used to illustrate different analytic approaches. The purpose was to compare analytic approaches and not to provide an analysis of a particular data set. Under this model, short term health-stratum-specific estimates are unbiased for estimating the health-stratumspecific instantaneous mortality hazard ratios from obesity, and updating information on body mass index and disease status during long term follow-up reduces bias. For followup over 10 or 20 years, exclusion of participants with preexisting disease, excluding the first five years of follow-up, and methods of analysis that ignore health status yield biased estimates of the instantaneous mortality hazard ratios. However, over 10 or 20 year time periods, long-term average mortality hazard ratios or cumulative mortality relative risks are a better reflection of the total impact of obesity, including its tendency to accelerate transitions to sickness under this model, than are instantaneous mortality hazard ratios. Over these longer time periods, average relative hazard estimates or cumulative mortality relative risks based on initially well participants, on initially sick participants, and on the combined initial population each provide valuable descriptions of associations of obesity with mortality.

KEYWORDS AND PHRASES: Causation, Markov chain, Mortality, Obesity, Reverse causation.

\footnotetext{
* Corresponding author.

${ }^{\dagger}$ This work was supported in part by the the Natural Sciences and Engineering Research Council of Canada (NSERC).

‡This work was supported in part by the Intramural Research Program of the National Cancer Institute.

$\S$ The findings and conclusions in this report are those of the authors and not necessarily those of the Centers for Disease Control and Prevention and the National Cancer Institute.
}

\section{INTRODUCTION}

For decades researchers have studied the association between obesity and mortality [14]. In these studies, obesity is usually measured by the body mass index (BMI), which is defined as body weight in kilograms divided by the square of height in meters. Yet there remain questions as to the uses and interpretation of the association between BMI and mortality, as well as how to estimate it. Several issues contribute to these questions. First, BMI is not a quantity that is controlled by the investigator's intervention, but rather an endogenous characteristic of the participant. Causal interpretations require that one can imagine an outcome for each participant in the presence and absence of an intervention. Therefore Hernan et al. [9] question whether there is a valid causal interpretation of the association. Second, BMI can change over time in response to lifestyle changes and health status, which may also be associated with the risk of death. This confounds the interpretation of the association, as discussed in detail in Robins [15], who proposes complex analyses to untangle causal effects. Third, weight and height are usually measured or self-reported only at the time of recruitment into the cohort. Even if one is interested in the modest goal of describing the association, without imposing a causal interpretation, the fact that BMI changes over time can make the association with baseline BMI a poor description of its effects over time.

A frequently reported feature of plots of mortality relative risk against BMI is that mortality relative risk attains a minimum near $\mathrm{BMI}=25$, with increases in relative risk for higher BMIs and for lower BMIs. This J- or U-shaped locus has caused some to question whether the higher mortality at low BMI reflects "reverse causation", namely the effects of pre-existing disease that decreases BMI and increases the risk of mortality $[13,18]$. This is the type of confounding addressed by Robins [15]. A number of simpler methods have been proposed to address this type of possible bias from pre-existing disease, including: (i) deleting the first few years of follow-up to eliminate deaths that may be due to illness present at baseline [1, 13]; and (ii) deleting study participants who are ill at baseline [13]. In this paper we address whether these maneuvers remove this type of bias in the context of a simple Markov model. We also study the effects of having repeated BMI and health sta- 
tus measurements over the course of follow-up in attaining unbiased estimates of BMI relative risk in this model.

We consider a simple Markov model with states defined by BMI category: Non-obese (BMI $\leq 30)$ and Obese (BMI $>30$ ); and by health status (Well, Sick, or Death) (Figure 1). A person migrates among these states over time. The process is completely described by the transition probabilities between each pair of states. The ratios of these probabilities define the relative risks of interest. For illustration, we use parameters for our model that were estimated from data from the first National Health and Nutrition Examination Survey Epidemiologic Follow-Up Study (NHEFS) [3] to demonstrate biases arising from different analytic strategies. To estimate transition probabilities from the NHEFS data, we defined Sick by the self-reported diagnosis of any of the following diseases: diabetes, high blood pressure, cancer, heart attack and stroke. All other live participants were considered as Well. Even though our model is simplified in many respects, the parameter values are not unrealistic, and the model captures many of the features that can confound more conventional analyses. Our model gives insight into biases that can afflict conventional analyses when the endogenous exposure (obesity) and confounders (health status) vary with time in these specified ways.

In the context of the Markov model, certain ratios of transition rates determine the effects of obesity on health outcome, and we therefore study how well various analytic strategies estimate these ratios. For example, if the mortality hazard ratio comparing Obese to Non-obese participants is the same for Sick and Well participants, this ratio is a parameter of great interest. However, over a long period of follow-up, the effect of obesity on mortality may be mediated by a faster rate of transition to the Sick state among Obese participants than among the Non-obese participants. Thus we may also be interested in the average mortality hazard ratio or the cumulative mortality relative risk over various follow-up periods, comparing those initially obese to those who are not obese. Such measures depend in a complex way on the parameters of the Markov model and on the duration of follow-up. We use this model to evaluate several analytic strategies to reduce bias from reverse causation and from changing BMI levels. We assume that the only information on each cohort member is the fourfold classification on BMI and health status at baseline and the subsequent date of death if any. For some analyses, we assume that this fourfold classification is available at one or more follow-up times. The analytic strategies we investigate are: use all participants (Method C); use only the Well participants (Method W); use only the Sick participants (Methods S); and exclude the mortality experience during the first 5 years (Method E). We also study the effects of varying the duration of follow-up, because it has been recommended that follow-up not extend beyond 15 years [18]. Finally, we consider the value of additional data on health and BMI status at various points during follow-up.

452 B. E. Chen et al.

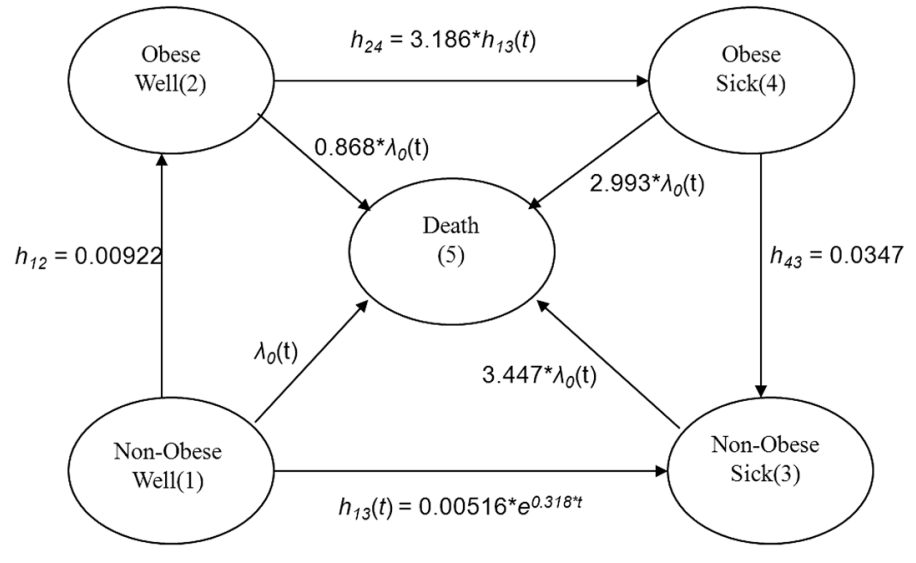

Figure 1. Graph of the Markov model showing estimated annual transition rates obtained from the NHEFS. The quantity $\lambda_{0}(t)=a \exp (b t)$ is the hazard of mortality for a Non-obese Well subject at age $t$, where $a=0.000088$ and $b=0.065$. The quantity $h_{13}(t)=c_{0} e^{c_{1} t}$ is the transition rate from Non-obese Well to Non-obese Sick participant at age $t$, where $c_{0}=0.00516$ and $c=0.0318$. The quantity $h_{24}(t)=c_{1} h_{13}(t)$ is the transition rate from Obese Well to Non-obese Sick subject at age $t$, where $c_{1}=3.186$.

\section{METHODS}

\subsection{The Markov model}

We use a first order discrete Markov Chain model where age of the participant $(t=0,1,2, \ldots)$ is chosen as the time scale. The combinations of two BMI states (Non-obese and Obese) and two health states (Well and Sick) give four different live states: Non-obese Well as state 1, Obese Well as state 2, Non-obese Sick as state 3 and Obese Sick as state 4. Finally, we define an absorbing state Death as the fifth state. Figure 1 shows the transition directions and rates among these five states based on data from the NHEFS. We limit the transitions among different states as follows. Except for the risks of mortality from each of the four live states with an age-specific mortality rate, participants can only move from the Non-obese Well (state 1) to Obese Well (state 2) at a constant annual rate $h_{12}$ or to Non-obese Sick (state 3 ), with age-specific rate $h_{13}(t)=c_{0} e^{c t}$ with $c_{0}>0$ and $0<h_{13}(t)<1$. Participants in the Obese Well (state 2 ) state will eventually die or become Obese Sick (state 4) with agespecific rate $h_{24}(t)=c_{1} h_{13}(t), c_{1}>0$, which is proportional to the transition probability from Non-obese Well to Nonobese Sick. Participants in the Obese Sick (state 4) could lose weight and become Non-obese Sick (state 3 ) with rate $h_{43}$, where $0<h_{43}<1$. We further assume that all of these moves are irreversible. We use the exponential function to model the mortality rates [8], assuming that participants in state 1 (Non-obese $W e l l$ ) have a baseline mortality rate at age $t$ :

$$
\lambda_{0}(t)=a \exp (b t)
$$


We assume that the relative mortality hazard between an Obese Well and a Non-obese Well individual is a constant $r_{21}$ and the relative mortality hazard between a Non-obese Sick and a Non-obese Well individual is a constant $r_{31}$. We assume a multiplicative relative risk model $r_{41}=r_{21} r_{31}$ to describe the relative mortality rate of Obese Sick to Nonobese Well. We use this assumption in numerical studies and to estimate parameters. However, this multiplicative assumption was relaxed when we investigated different analytic strategies. Thus the age-specific transition probability matrix at age $t$ is given by,

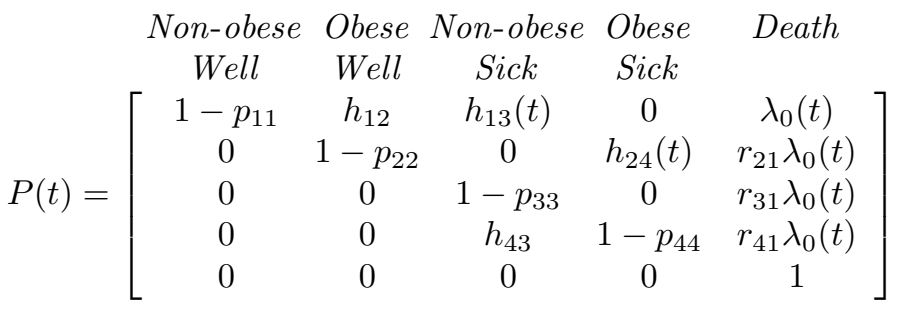

where $p_{i i}=\sum_{j \neq i} p_{i j}(t)$ and $p_{i j}(t)$ is the $(i, j)$ entry of matrix $P(t)$. For any integer $k \geq 1$, starting at age $t$, the $k$-step transition matrix can be written as

$$
P^{(k)}(t)=\prod_{s=1}^{k} P(t+s-1),
$$

where $P^{(0)}(t)=I$. Let $p_{i j}^{(k)}(t)$ be the $(i, j)$ entry of matrix $P^{(k)}(t)$.

Participants in the NHEFS were assessed for BMI and health states at two examinations about ten years apart. All participants were followed-up for mortality status. Not all respondents at baseline participated in the second examination. For participant $i$, we let $\left\{a_{1 i}, a_{2 i}, y_{1 i}, y_{2 i}, v_{i}, t_{i}, \delta_{i}\right\}_{i=1}^{n}$ be a set of observation vectors, where $a_{1 i}$ is the age at enrollment, $a_{2 i}$ is the age at the second exam and $t_{i}$ is the age that participant $i$ dies or is last seen alive. Let $y_{1 i}$ and $y_{2 i}$ be the states at the enrollment and the second exam for participant $i$, respectively. Let $\delta_{i}=1$ if participant $i$ dies at time $t_{i}$, and $\delta_{i}=0$ otherwise. Let $v_{i}$ be an indicator variable with $v_{i}=1$ if participant $i$ got a second exam and $v_{i}=0$ otherwise. In case participant $i$ did not get a second exam, both $a_{2 i}$ and $y_{2 i}$ are assumed to be missing completely at random [12].

Let $\theta=\left(a, b, c, c_{0}, c_{1}, h_{24}, h_{43}, r_{21}, r_{31}\right)$ denote the unknown parameter vector. The log likelihood function for $n$ participants with $\left\{a_{1 i}, a_{2 i}, y_{1 i}, y_{2 i}, v_{i}, t_{i}, \delta_{i}\right\}_{i=1}^{n}$ is

$$
\begin{aligned}
\ell(\theta)= & \sum_{i=1}^{n} v_{i} \log \left\{p _ { y _ { 1 i } , y _ { 2 i } } ^ { a _ { 2 i } - a _ { 1 i } } ( a _ { 1 i } ) \left(\delta_{i} p_{y_{2 i}, 5}^{t_{i}-a_{2 i}}\left(a_{2 i}\right)\right.\right. \\
& \left.\left.+\left(1-\delta_{i}\right) \sum_{j=1}^{4} p_{y_{2 i}, j}^{t_{i}-a_{2 i}}\left(a_{2 i}\right)\right)\right\} \\
& +\sum_{i=1}^{n}\left(1-v_{i}\right) \log \left\{\delta_{i} p_{y_{1 i}, 5}^{t_{i}-a_{1 i}}\left(a_{1 i}\right)\right.
\end{aligned}
$$

$$
\left.+\left(1-\delta_{i}\right) \sum_{j=1}^{4} p_{y_{1 i}, j}^{t_{i}-a_{1 i}}\left(a_{1 i}\right)\right\} .
$$

Maximum likelihood estimators $\hat{\theta}$ were obtained by applying fmincon, the non-linear optimization procedure with constraints in MATLAB (The MathWorks Inc., 2010) to the NHEFS dataset of $n=14,407$. The initial value is given by $\theta_{0}=(-4,0.01,0.01,-3,1,0.05,0.05,1,1)$, with parameters $a$ and $c_{0}$ given on the logarithmic scale. The constraints on the parameters are given by the lower boundary $\theta_{\text {lower }}=(-20,-0.1,-0.1,-20,1,0,0,0,0)$, and the upper boundary $\theta_{\text {upper }}=(0,0.1,0.1,0,20,0.2,0.2,20,20)$. Several other initial values were used with this procedure and the same maximum likelihood estimates were obtained as long as the log likelihood function $\ell\left(\theta_{0}\right)$ was finite. Because NHEFS data are used here only to motivate the choice of model parameters, survey sample weights were not applied in this calculation. The corresponding estimate of the transition matrix is denoted $\hat{P}(t)$.

Let $N(t)=\left\{n_{1}(t), n_{2}(t), n_{3}(t), n_{4}(t)\right\}^{\prime}$ be the total number of participants alive at age $t$ in states $1,2,3$ and 4 (Figure 1). We assume that the number $N(35)$ is known at the starting age $t=35$. For any $t>35, E\{N(t)\}=$ $E\{N(t-1)\} P(t-1)$. Thus, using the Markov model, we can generate the expected numbers of individuals in each of the living states at subsequent ages.

\subsection{Measures of the effects of obesity on mortality}

Analysts often use the proportional hazards model to estimate health effects. Even if hazards are not truly proportional, this analysis yields an average hazard ratio. To mimic this approach, we use another average hazard ratio proposed by Tarone [17]. Consider the hazard ratio comparing a population of Obese participants at age $t$ with a population of Non-obese participants at age $t$ who are followed for the next $F$ years. Let $m_{2}(t)=n_{2}(t)+n_{4}(t)$ be the total number of the Obese Well and Obese Sick participants and $m_{1}(t)=n_{1}(t)+n_{3}(t)$ be the total number of Non-obese Well and Non-obese Sick participants. The Obese groups will go through complex transitions among live states but will yield deaths $d_{2 j}(t)$ for $j=1,2, \ldots, F$ in each of the next $F$ years, whereas the Non-obese groups will yield $d_{1 j}(t)$ deaths in each succeeding year. Let $m_{1 j}(t)$ and $m_{2 j}(t)$ denote the numbers of Non-obese and Obese participants respectively who are still at risk at the beginning of the $j$ th year after entry on study at age $t$. The hazard ratio estimate for the $j$ th year is

$$
\hat{r}_{j}(t)=\frac{d_{2 j}(t) / m_{2 j}(t)}{d_{1 j}(t) / m_{1 j}(t)} .
$$

The exact values of $m_{1 j}(t)$ and $m_{2 j}(t)$ are unknown when the dataset is not updated annually. However, assuming that the only losses from the population are from deaths and that 
Table 1. Maximum likelihood estimates of parameters from NHANES-I epidemiologic follow-up data on 14,407 participants

\begin{tabular}{|c|c|c|c|c|c|}
\hline & & Parameter & S.E. & Lower $95 \%$ CI & Upper $95 \%$ CI \\
\hline \multicolumn{6}{|l|}{ Transition Rates } \\
\hline Non-obese Well to Obese Well, & $h_{12}$ & 0.00922 & 0.000285 & 0.00866 & 0.00978 \\
\hline \multirow{2}{*}{ Non-obese Well to Non-obese Sick $h_{13}(t)=c_{0} e^{c t}$} & $c_{0}$ & 0.00516 & 0.00297 & 0.00461 & 0.00578 \\
\hline & $c$ & 0.0318 & 0.00102 & 0.0298 & 0.0338 \\
\hline Obese Well to Obese Sick $h_{24}(t)=c_{1} h_{13}(t)$ & $c_{1}$ & 3.186 & 0.0983 & 2.993 & 3.379 \\
\hline Obese Sick to Non-obese Sick & $h_{43}$ & 0.0374 & 0.00187 & 0.0337 & 0.0411 \\
\hline \multicolumn{6}{|l|}{ Mortality Hazards and Hazard Ratios } \\
\hline \multirow[t]{2}{*}{ Non-obese Well $(a \exp (b t))$} & $a$ & 0.000088 & 0.000007 & 0.000075 & 0.000103 \\
\hline & $b$ & 0.0650 & 0.00112 & 0.0629 & 0.0672 \\
\hline Obese Well to Non-obese Well & $r_{21}$ & 0.868 & 0.0436 & 0.782 & 0.953 \\
\hline Non-obese Sick to Non-obese Well & $r_{31}$ & 3.447 & 0.210 & 3.036 & 3.859 \\
\hline Obese Sick to Non-obese Well & $r_{21} r_{31}$ & 2.993 & $0.221^{\dagger}$ & 2.590 & 3.458 \\
\hline
\end{tabular}

$\dagger$ Standard error (S.E.) and 95\% confidence interval (CI) for $r_{21} r_{31}$ are based on the logarithmic transformation log $\left(r_{21} r_{31}\right)$, use of the delta method to get its variance,taking $\operatorname{Cov}\left(\log \left(r_{21}, r_{31}\right)\right)$ into account and then transforming the CI of $\log \left(r_{21} r_{31}\right)$ back to a CI for $r_{21} r_{31}$.

all deaths are recorded, we set $m_{1 j}(t)=m_{1}(t)-\sum_{k=1}^{j-1} d_{1 r}(t)$ and $m_{2 j}(t)=m_{2}(t)-\sum_{k=1}^{j-1} d_{2 r}(t)$ to approximate the number of participants that are still at risk. There will be $F$ such hazard ratios $\hat{r}_{j}(t)$ for follow-up years $j=1,2, \ldots, F$. If a proportional hazards model had been fitted to these data, a weighted average of these $F$ hazard ratios would be produced as the estimated hazard ratio. Rather than use the proportional hazards model, we use a summary estimate of the relative hazard from (author?) [17]. In particular, we set

$$
\hat{r}_{21}^{(C)}(t, F)=\frac{\sum_{j=1}^{F} W_{j}(t) \hat{r}_{j}(t)}{\sum_{j=1}^{F} W_{j}(t)}
$$

where

$$
W_{j}(t)=\frac{m_{1 j}(t) m_{2 j}(t)}{m_{\cdot j}(t)} \times \frac{d_{\cdot j}(t)}{m_{\cdot j}(t)-d_{\cdot j}(t)}
$$

and where $m_{\cdot j}(t)=m_{1 j}(t)+m_{2 j}(t)$ and $d_{\cdot j}(t)=d_{1 j}(t)+$ $d_{2 j}(t)$. For each fixed $F$ such as $F=1,5,10,20$ years, we plotted $\hat{r}_{21}^{(C)}(t, F)$ as a function of age $t$. The superscript $C$ indicates that the Sick and Well participants are combined in this comparison of Obese with Non-obese participants.

Another measure of the summary effect of obesity that has clinical appeal is the cumulative mortality ratio. We define the cumulative mortality ratio after $F$ years of followup for the combined Sick and Well participants as

$$
c \hat{m} r^{(C)}(t, F)=\frac{\sum_{j=1}^{F} d_{2 j}(t) / m_{2}(t)}{\sum_{j=1}^{F} d_{1 j}(t) / m_{1}(t)} .
$$

We also estimated hazard ratios from subsets of the population. Using only the Well participants (Method W), we estimated the hazard ratio $\hat{r}_{21}^{(W)}(t, F)$ and the cumulative mortality ratio $c \hat{m} r^{(W)}(t, F)$ by the previous formulas but used Obese Well participants instead of all Obese participants and Non-obese Well participants instead of all Nonobese participants. Similarly, using only the Sick participants (Method $\mathrm{S}$ ), we estimated the hazard ratio $\hat{r}_{21}^{(S)}(t, F)$ and the cumulative mortality ratio $c \hat{m} r^{(S)}(t, F)$ with Obese Sick participants instead of all Obese participants and with Non-obese Sick participants instead of all Non-obese participants.

To examine the effects of excluding deaths during the first five years, we used the previous formulas but computed summary estimates of relative risk by summing only over years of follow-up $j=6,7, \ldots, F$.

We are interested in short term and long term measures of the effects of obesity on mortality. Short term health stratum-specific estimates of the mortality hazard ratios from obesity are unbiased for $r_{21}$, but other estimates are not. Over longer periods of follow-up, the effects of obesity cannot be summarized in a single parameter of the Markov model. We therefore calculated average hazard ratios and cumulative mortality ratios as described above to compare Obese to Non-obese participants either in the whole population or among initially Sick participants or among initially Well participants as a function of age at beginning of followup and follow-up duration. Analysts who are most interested in the comparison among initially well participants might regard $\hat{r}_{21}^{(W)}(t, F)$ or $c \hat{m} r^{(W)}(t, F)$ as the quantities of interest. Analysts interested in the association of obesity with mortality in the entire population might regard $\hat{r}_{21}^{(C)}(t, F)$ or $c \hat{m} r^{(C)}(t, F)$ as primary.

Bias of relative risks for short-term follow-up analysis is relative to the true relative mortality hazards ratios $r_{21}$ and $r_{31}$. For long-term follow-up, $r_{21}$ and $r_{31}$ no longer capture the full effect of obesity because of transitions among the live states. Therefore, we do not use the term bias for long term projections but use curves (for example in Figure 3a) to describe the effects of obesity on entire population. 


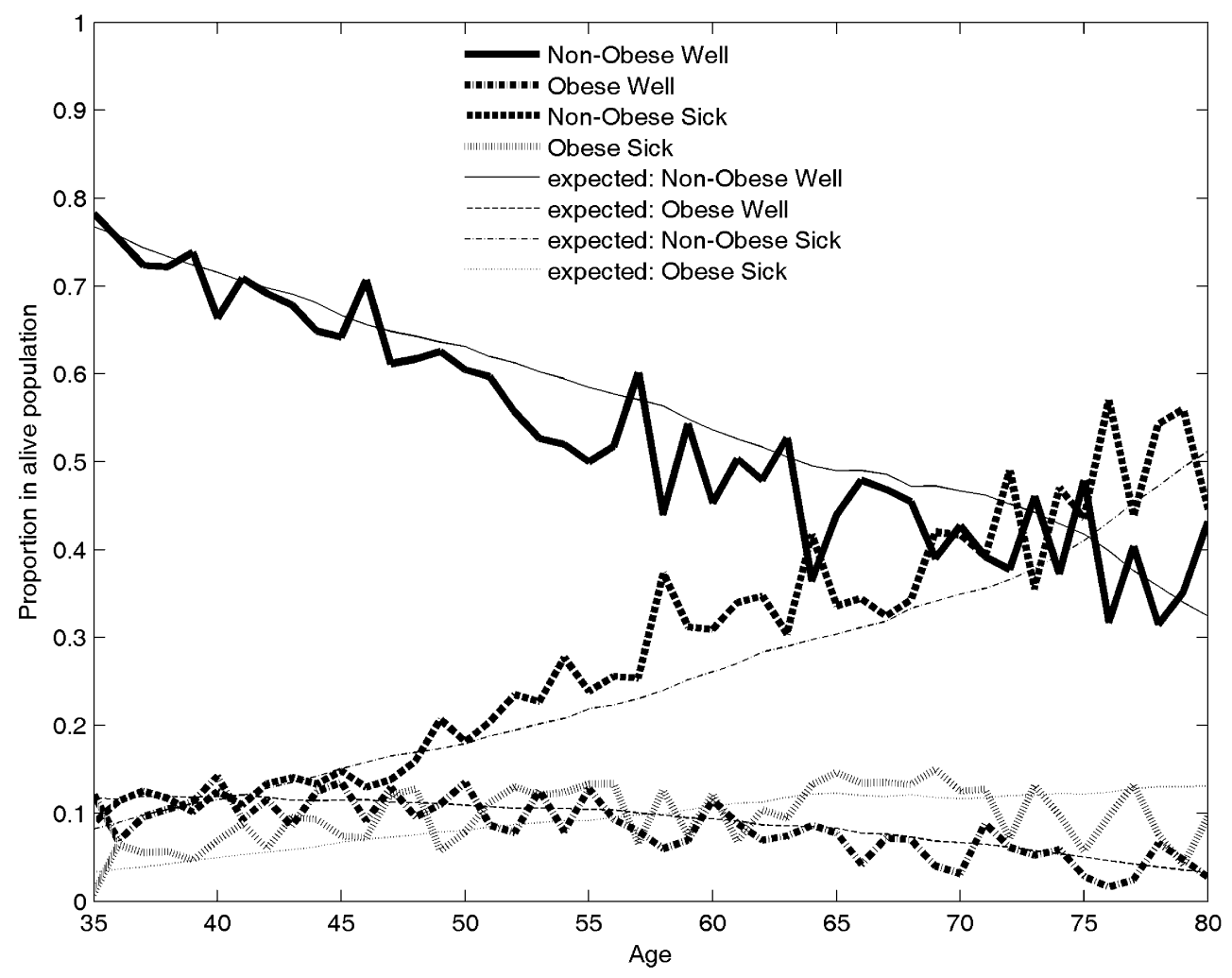

Figure 2. The empirical (from the NHEFS second exam) and fitted (from the parameters in Table 1) proportions in various live states for ages 35 to 85 .

\section{RESULTS}

\subsection{Obtaining parameter estimates for the Markov model}

Ten maximum likelihood estimates from NHEFS data are given in Table 1, together with confidence intervals. Only nine of these estimates represent functionally independent parameters. To check the model, we plotted the proportions of participants in the various live states estimated from the second examination in the NHEFS against age and compared them with model-based estimates. These latter estimates are the expected proportions by age at follow-up for a hypothetical population of individuals who are 35 years old at baseline, with the same baseline health status distribution as the 35-year-old in the first examination in NHEFS (Figure 2). The model captures the qualitative features of the data.

The baseline mortality rate for Non-obese Well and the transition probabilities between the four live states from model (2.2) were used in what follows to examine the various analytic strategies. That is, all seven parameters $a, b, c, c_{0}, c_{1}, h_{24}, h_{43}$ stay constant in rest of paper, while mortality hazard ratios for Obese Well versus Non-obese well $r_{21}$ and Obese Sick versus Non-obese Sick $r_{31}$ vary in subsequent numerical studies.

\subsection{Comparing obese to non-obese participants}

Under the unrealistic null hypothesis of no effect of obesity or sickness on mortality, $H_{N \text { ull }}: r_{21}=r_{31}=1$, each of the estimates $\hat{r}_{21}^{(W)}, \hat{r}_{21}^{(S)}$ and $\hat{r}_{21}^{(C)}$ (to simplify the notation, we drop $(t, F)$ for both $\hat{r}_{21}$ and $\left.c \hat{m} r\right)$ provides unbiased results for $r_{21}=1$ at all ages and for follow-up times 1, 5, 10 and 20 years (data not shown). Now suppose $r_{21}=1$ and $r_{31}=2$ (Figure 3a). Then the effect of obesity is only through its influence on the rate of transition to sickness. For one year of follow-up, both $\hat{r}_{21}^{(W)}$ and $\hat{r}_{21}^{(S)}$ estimate $r_{21}=1$ correctly, but $\hat{r}_{21}^{(C)}$ is upwardly biased because the Obese and Non-obese populations are mixtures of Well and Sick participants. For older participants the bias in $\hat{r}_{21}^{(C)}$ is less, because most of the older participants become sick. Over longer periods of follow-up, the behavior becomes more complex. For $F=5,10$ and 20, $\hat{r}_{21}^{(C)}$ exceeds $r_{21}=1$ because Obese Well participants become Obese Sick participants over time. Interestingly, using only sick participants as in strategy $\mathrm{S}$ yields realistic estimates $\hat{r}_{21}^{(S)}$ for $r_{21}=1$ for $F=5,10$ and 20, because patients cannot revert from a sick to a well state in our model. For $F=5,10$ and 20, the combined estimate $\hat{r}_{21}^{(C)}$ is larger than $\hat{r}_{21}^{(W)}$ for young participants and smaller than $\hat{r}_{21}^{(W)}$ for older participants, who have a higher preva- 

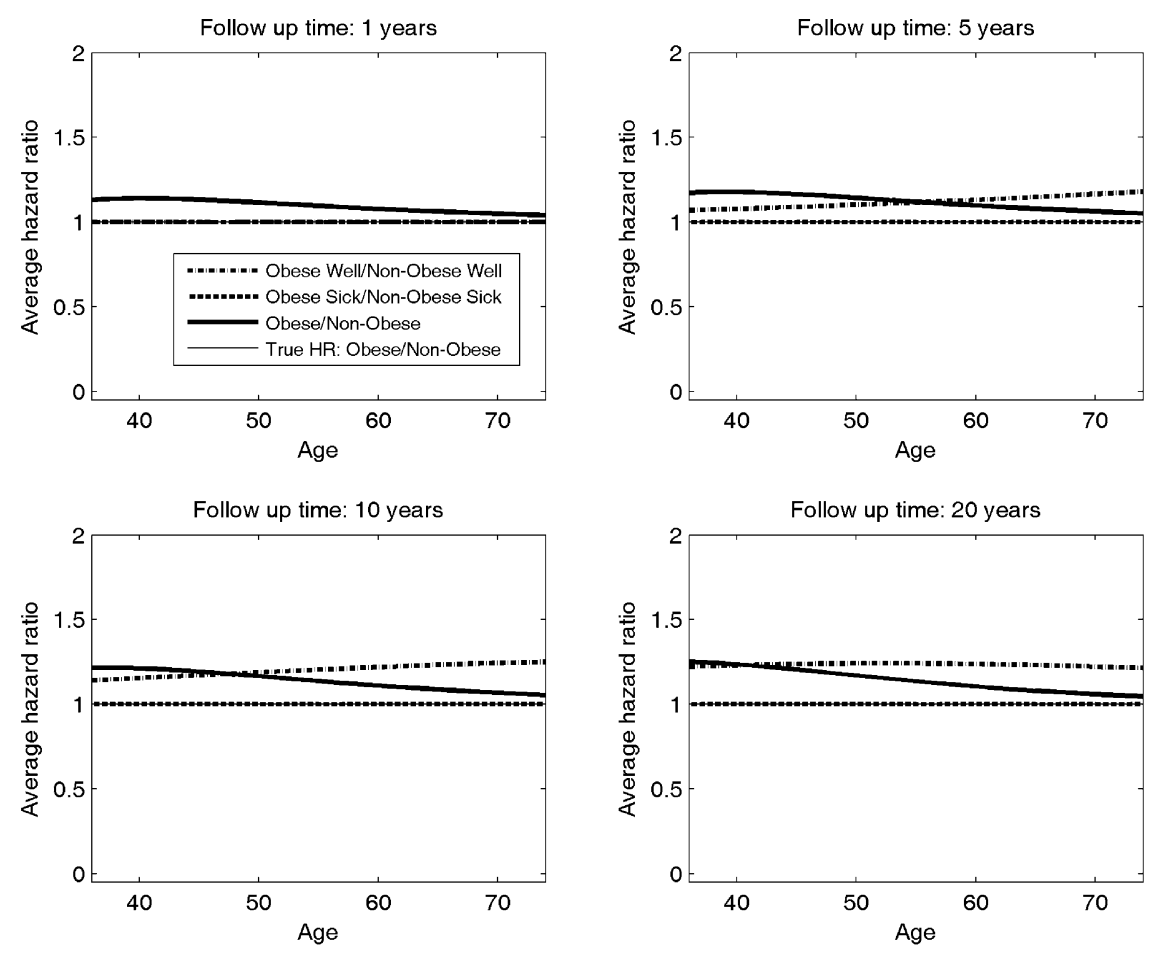

Figure 3a. Estimated mortality hazard ratios comparing Obese to Non-obese participants. The $x$-axis is the ages at baseline. The multiplicative model is assumed under which the mortality hazard ratio for Obese Sick compared to Non-obese Well is $r_{41}=r_{21} r_{31}$. The hazard ratio $r_{21}=1$ compares Obese Well to Non-obese Well, and, under the multiplicative model also compares Obese Sick to Non-obese Sick. The hazard ratio $r_{31}=2$ compares Non-obese Sick to Non-obese Well, and, under the multiplicative model also compares Obese Sick to Obese Well. Estimators based on Well participants only, $\hat{r}_{21}^{(W)}$, on Sick participants only, $\hat{r}_{21}^{(S)}$, and on Sick and Well participants combined, $\hat{r}_{21}^{(C)}$, were studied.
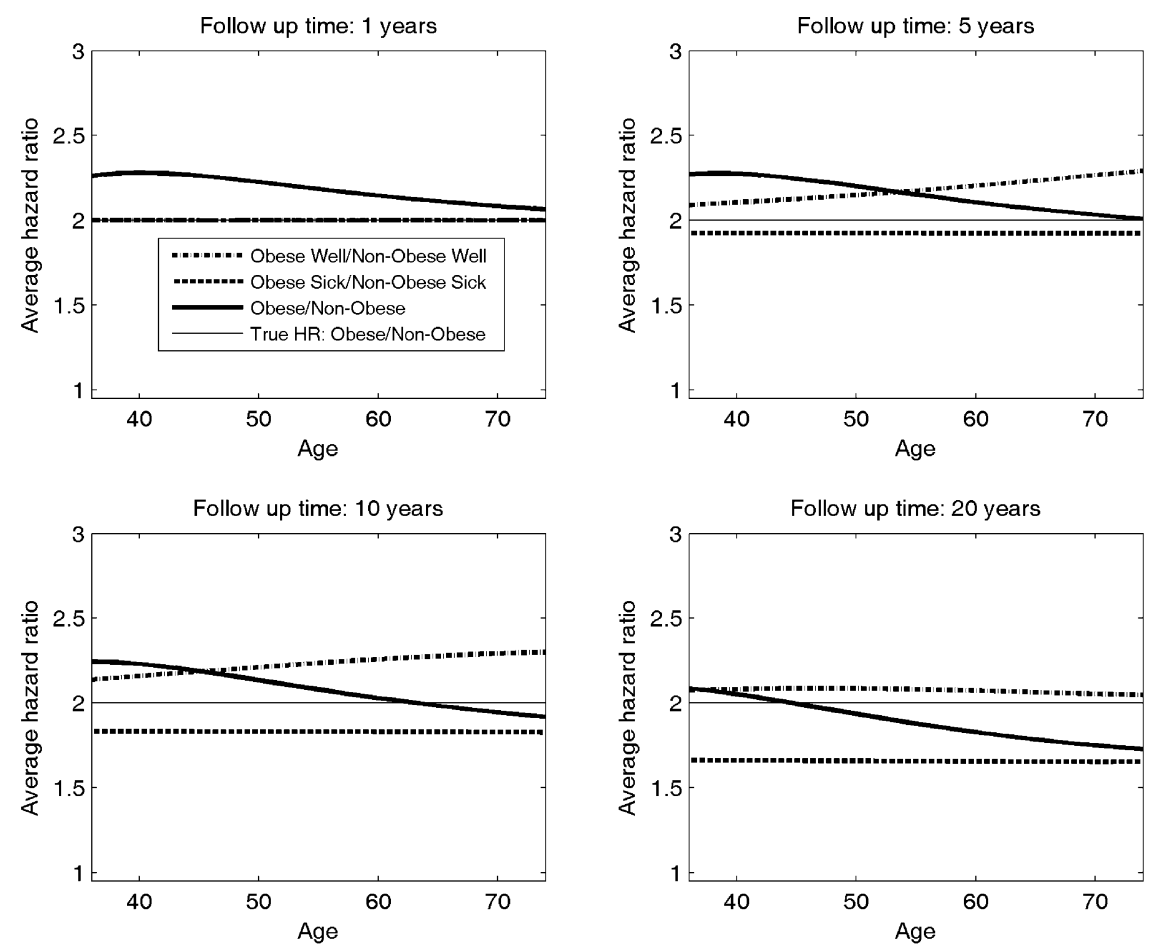

Figure 3b. Estimated mortality hazard ratios comparing Obese to Non-obese participants. The $x$-axis is the ages at baseline. The hazard ratios $r_{21}=2, r_{31}=2$ and $r_{41}=4$.

456 B. E. Chen et al. 

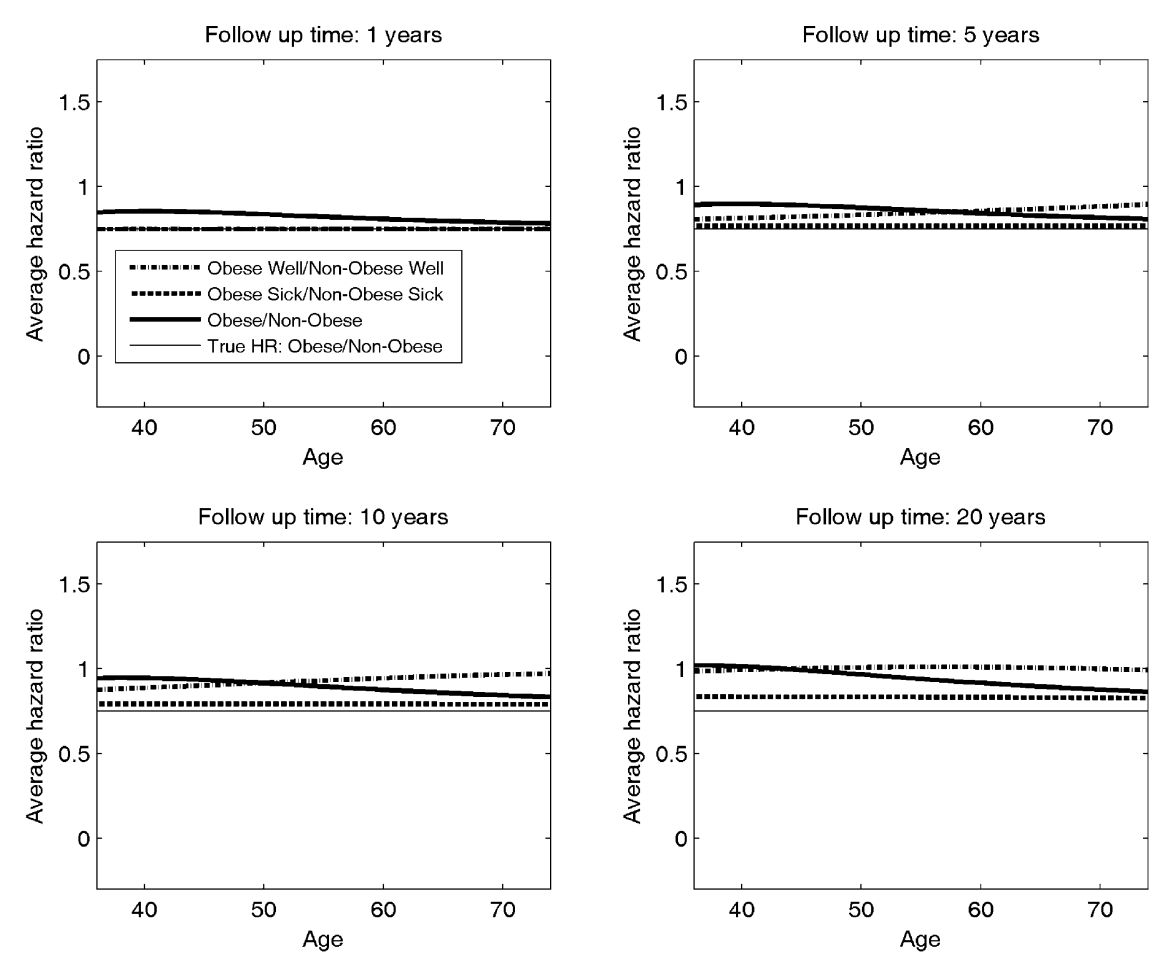

Figure 3c. Estimated mortality hazard ratios comparing Obese to Non-obese participants. The $x$-axis is the ages at baseline. The hazard ratios $r_{21}=0.75, r_{31}=2$ and $r_{41}=1.5$.
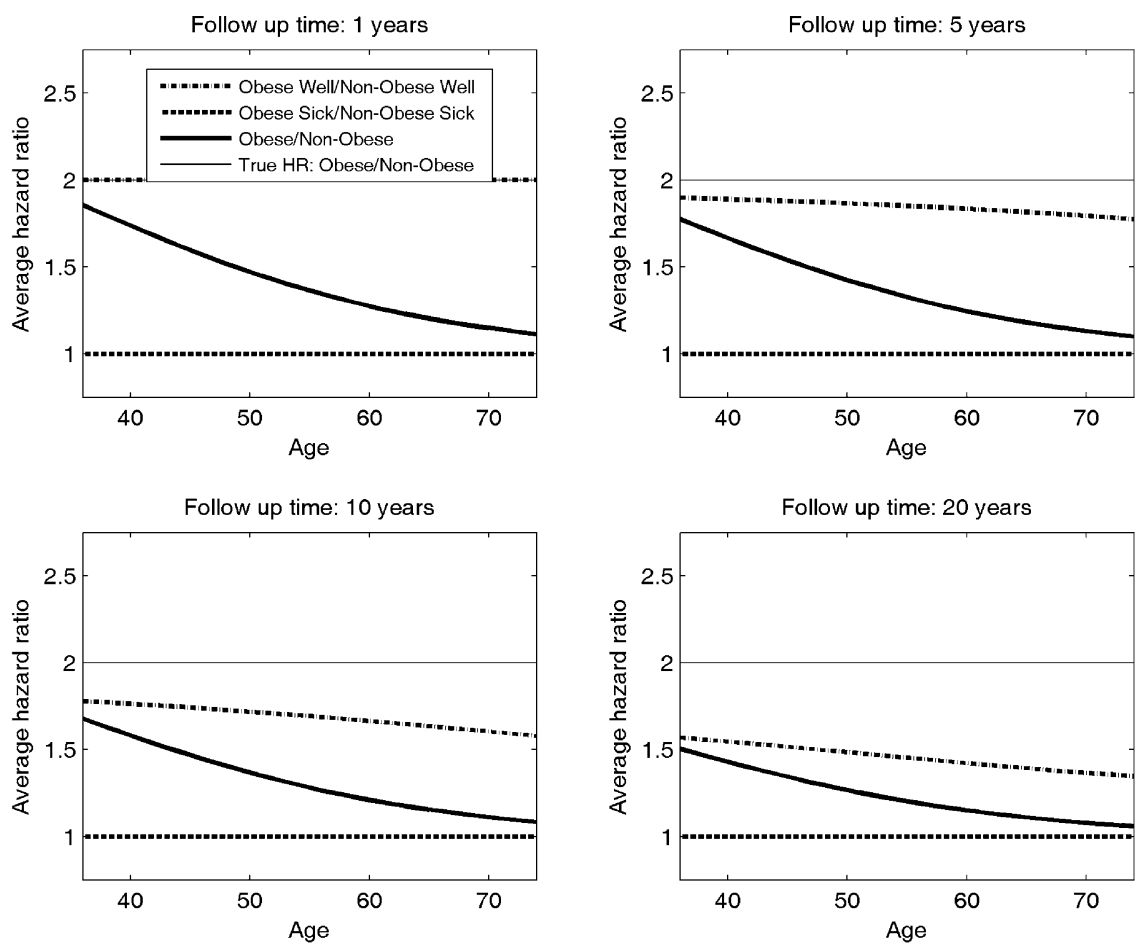

Figure 3d. Estimated mortality hazard ratios comparing Obese to Non-obese participants. The $x$-axis is the ages at baseline. The hazard ratios $r_{21}=2, r_{31}=2$ and $r_{41}=2$. Thus the mortality hazard ratio for Obese to Non-obese is 2 for Well and 1 for Sick participants. 
lence of sickness. For follow-up periods $F=5,10$ and 20, some analysts would regard $\hat{r}_{21}^{(W)}$ as giving the most realistic assessment of the effect of obesity, as it allows for the effect of transitions to sickness in a population initially unconfounded by sickness. Others might be more interested in $\hat{r}_{21}^{(C)}$ as a measure of the association of obesity with mortality in the entire population. Similar comments apply to cumulative mortality ratios $c \hat{m} r^{(W)}, c \hat{m} r^{(S)}$ and $c \hat{m} r^{(C)}$ as to $\hat{r}_{21}^{(W)}, \hat{r}_{21}^{(S)}$ and $\hat{r}_{21}^{(C)}$, as shown in Figure 4 .

If $r_{21}=2$ and $r_{31}=2$, obesity influences the rate of mortality by its direct effect on the mortality hazard in Obese Well and Obese Sick participants, and also because it accelerates the transition to sickness (Figure $3 \mathrm{~b}$ ). The qualitative conclusions in this case are similar to those in Figure 3a. For $F=1, \hat{r}_{21}^{(W)}$ and $\hat{r}_{21}^{(S)}$ are unbiased for $r_{21}=2$, but $\hat{r}_{21}^{(C)}$ is upwardly biased. For $F=5$ or 10 years, $\hat{r}_{21}^{(W)}$ exceeds $r_{21}=2$, $\hat{r}_{21}^{(S)}$ underestimates $r_{21}=2$ and $\hat{r}_{21}^{(C)}$ exceeds $r_{21}=2$ at young but not old ages. For $F=20$ years, $\hat{r}_{21}^{(W)}, \hat{r}_{21}^{(S)}$ and $\hat{r}_{21}^{(C)}$ underestimate $r_{21}=2$, except at the youngest ages. For $F=20$ years, $\hat{r}_{21}^{(W)}$ exceeds $\hat{r}_{21}^{(C)}$ at most ages. If $\hat{r}_{21}^{(W)}$ is thought to reflect the biological impact of obesity, then $\hat{r}_{21}^{(C)}$, which describes the association with obesity in the entire population, underestimates the biologic impact. $\mathrm{Cu}$ mulative mortality ratios yield similar patterns as in Figure 3b (unreported data).

If instead $r_{21}=0.75$ and $r_{31}=2$ (Figure 3c), obesity has a protective effect on the mortality hazards, both for Obese Sick and Obese Well participants, but this protective effect is partly offset by the more rapid transition to sickness among Obese Well participants than among Non-obese Well participants. For $F=1$ year, $\hat{r}_{21}^{(W)}$ and $\hat{r}_{21}^{(S)}$ are unbiased for $r_{21}=0.75$, but $\hat{r}_{21}^{(C)}$ is upwardly biased. For $F=5,10$ and 20 years, $\hat{r}_{21}^{(W)}, \hat{r}_{21}^{(S)}$ and $\hat{r}_{21}^{(C)}$ exceed $r_{21}=0.75$, thereby underestimating the protective effect of obesity on mortality transition rates. For longer follow-up times, $\hat{r}_{21}^{(C)}$ exceeded $\hat{r}_{21}^{(W)}$ in young, but not old participants. Cumulative mortality ratios yield similar patterns as in Figure 3c (unreported data).

As is seen in Figures $3 \mathrm{a}, 3 \mathrm{~b}$ and $3 \mathrm{c}, \hat{r}_{21}^{(W)}$ and $\hat{r}_{21}^{(S)}$ yield unbiased estimates for $F=1$. It is not surprising, therefore, that if the information on health status and obesity status in living participants is updated each year, these estimators yield unbiased estimates, not only for $F=1$, but for $F=5,10$ and 20 years of follow-up (data not shown). Even if the data on obesity and health status are updated every year, the estimates $\hat{r}_{21}^{(C)}$ remain biased if $r_{31} \neq 1$ because $\hat{r}_{21}^{(C)}$ is not stratified on health status. Nonetheless, biases were reduced considerably if the information on health status and obesity status were updated during the follow-up. For example, for a 65 year old at baseline, with $r_{21}=2$ and $r_{31}=2$ and $F=20$ years of follow-up, the differences between $\hat{r}_{21}^{(W)}, \hat{r}_{21}^{(S)}$ and $\hat{r}_{21}^{(C)}$ and $r_{21}=2$ were reduced from $-0.254,-0.556$ and -0.463 without updating to $0.222,-0.121$ and -0.029 respectively with updating every five years. These latter biases are comparable to biases with $F=5$ years of follow-up.

Excluding the mortality experience during the first five years of follow-up does not move $\hat{r}_{21}^{(W)}, \hat{r}_{21}^{(S)}$ or $\hat{r}_{21}^{(C)}$ much closer to $r_{21}=2$ for $F=10$ or 20 years. For instance, for a 65 year old at baseline with $r_{21}=2$ and $r_{31}=2$ and $F=20$ years of follow-up, the differences between $\hat{r}_{21}^{(W)}, \hat{r}_{21}^{(S)}$ and $\hat{r}_{21}^{(C)}$ and $r_{21}=2$ changed from $-0.254,-0.556$ and -0.463 respectively with no exclusion to $-0.321,-0.660$ and -0.565 respectively with exclusion of deaths in the first five years of follow-up. Similar results were obtained for cumulative mortality ratios (not reported).

If obesity imposes less risk for a sick person than for a well person, then the multiplicative assumption $r_{41}=r_{21} \times r_{31}$ does not hold. In this case the risk from obesity depends on health status, and health status modifies the effect of obesity. Thus, no single parameter like $r_{21}$ characterizes effect of obesity on mortality transition rates. To study this situation with $r_{21}=2$ and $r_{31}=2$, we let $r_{41}=2$ instead of $r_{41}=r_{21} \times r_{31}=4$. Thus the relative mortality hazard from obesity is 2 for Well participants and 1 for Sick participants. For $F=1$ year, $\hat{r}_{21}^{(W)}$ and $\hat{r}_{21}^{(S)}$ provide unbiased estimates for the Obese to Non-obese relative risks in the Well population and in the Sick population, respectively (Figure 3d). With $F=5,10$ and 20 years, the estimate based on the Well population $\hat{r}_{21}^{(W)}$ underestimates $r_{21}=2$. However, $\hat{r}_{21}^{(S)}$, which is based on the Sick population, is a realistic estimate of the relative mortality hazard from obesity $(=1)$ for Sick participants, because our model does not allow for transitions from Sick to Well states. For the combined Well and Sick populations, the estimate $\hat{r}_{21}^{(C)}$ is nearer to $r_{21}=2$ in young participants and to $r_{41} / r_{31}=1$ in old participants (Figure $3 \mathrm{~d})$.

\section{DISCUSSION}

We used a Markov model that included four live states based on obesity status and health status to study analytical methods to assess associations of obesity with the risk of death. The model was specialized for chronic diseases because it did not allow transitions from sick to well states. Even this simple model illustrated several important aspects of analysis. There is an important analytical distinction between immediate effects of obesity and long-term effects. Under the Markov model and the multiplicative relationship $r_{41}=r_{21} \times r_{31}$, the parameter $r_{21}$ characterizes the immediate impact of obesity on mortality rates both in Well participants and in Sick participants. When $r_{21}=r_{31}=1$ each of the estimates $\hat{r}_{21}^{(W)}, \hat{r}_{21}^{(S)}$ and $\hat{r}_{21}^{(C)}$ provide unbiased results for $r_{21}$ at all ages and for follow-up times $F=1,5$, 10 and 20 years. The following discussion covers the cases when either $r_{21}$ or $r_{31}$ are not equal to 1 . To get unbiased estimates of $r_{21}$, one needs to stratify on health status (i.e. estimate with $\hat{r}_{21}^{(W)}$ or $\hat{r}_{21}^{(S)}$ or with $c \hat{m} r^{(W)}$ or $\left.c \hat{m} r^{(S)}\right)$ and use 

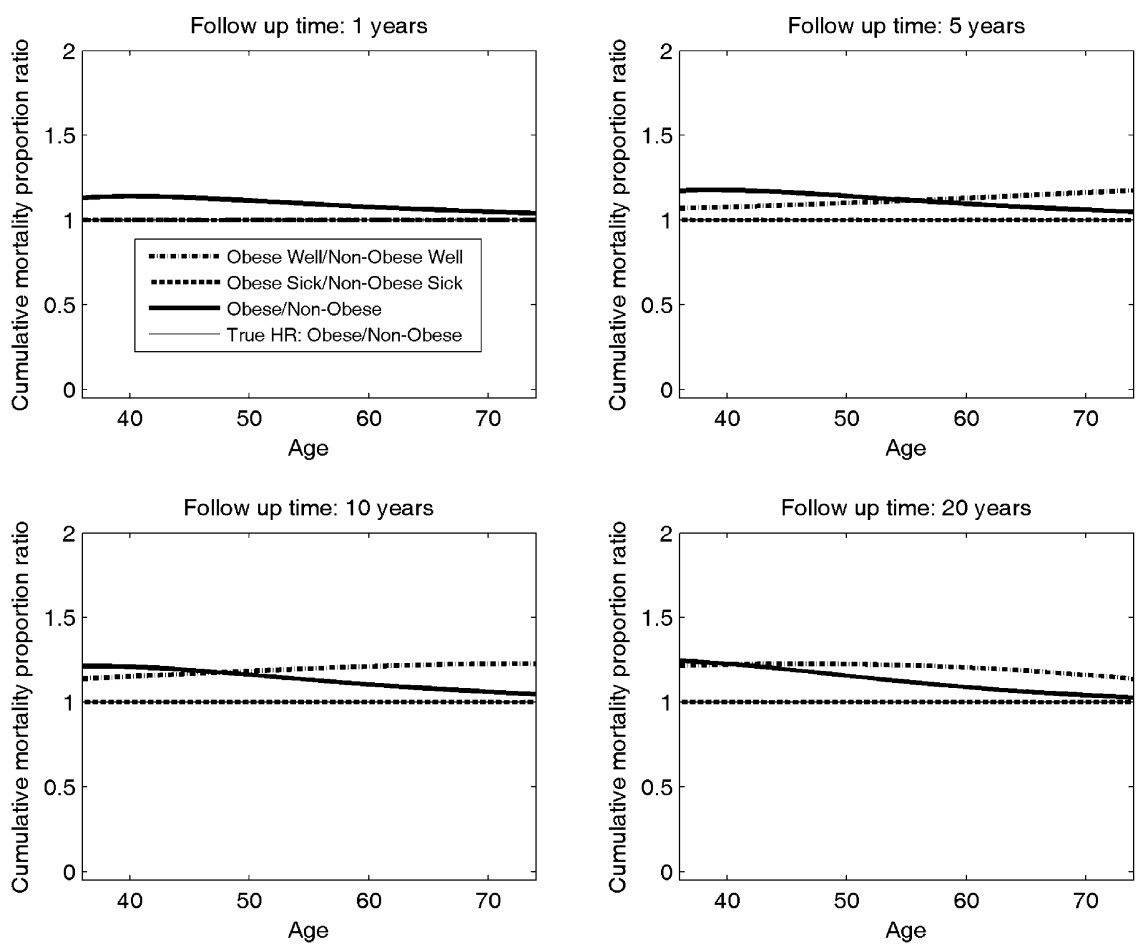

Figure 4. Estimated cumulative mortality ratios comparing Obese to Non-obese participants. The $x$-axis is the ages at baseline. The hazard ratio $r_{21}=1$ compares Obese Well to Non-obese Well, and, under the multiplicative model also compares Obese Sick to Non-obese Sick. The hazard ratio $r_{31}=2$ compares Non-obese Sick to Non-obese Well, and, under the multiplicative model $\left(r_{41}=r_{21} \times r_{31}=2\right)$ also compares Obese Sick to Obese Well. Estimators based on Well participants only, $c \hat{m} r_{21}^{(W)}$, on Sick participants only, $c \hat{m} r_{21}^{(S)}$, and on Sick and Well participants combined, $c \hat{m} r_{21}^{(C)}$, were studied.

short follow-up times such as $F=1$ year. A non-stratified estimate, $\hat{r}_{21}^{(C)}$, is biased. Good performance from stratified estimators can be obtained over longer follow-up periods if information on obesity and health status is updated periodically. However, studying participants who were Well initially $\left(\hat{r}_{21}^{(W)}\right.$ or $\left.c \hat{m} r^{(W)}\right)$ or Sick initially $\left(\hat{r}_{21}^{(S)}\right.$ or $\left.c \hat{m} r^{(S)}\right)$ does not yield unbiased estimates of the instantaneous mortality ratio $r_{21}$ over long follow-up periods, nor does eliminating mortality data from the first five years of follow-up.

Over longer follow-up periods such as $F=5,10$ or 20 years, several parameters in the Markov model are needed to characterize the effects of obesity. In particular, the parameter $c_{1}=3.186$ (Figure 1, Table 1) is important, as it determines the rate at which Obese Well participants transition to Obese Sick participants, compared to the rate at which Non-obese Well participants transition to Non-obese Sick participants. Over long periods, the effects of obesity on mortality are therefore partly mediated by increased rates of transition to sickness. In this context, no single Markov parameter describes the effect of obesity on mortality. Some analysts might take the average long term relative mortality hazard comparing initially Obese Well to initially Nonobese Well, $\hat{r}_{21}^{(W)}$ as a good summary measure of the effect of obesity on the initially healthy subject. For example, see
Manson et al. [13] and references therein. Others might be more interested in the long-term association of obesity with mortality in the entire population, estimated by $\hat{r}_{21}^{(C)}$, as in Flegal et al. [7]. Yet others might be interested in the long-term association of obesity with mortality among those who were initially sick, as measured by $\hat{r}_{21}^{(S)}$, because chronically ill participants are very prevalent in older segments of the population (Figure 2) and understanding associations of obesity with mortality among chronically ill participants is of public health importance. These three descriptors of the association of obesity with mortality can differ appreciably (Figures 3a-3d), yet all may be useful. The corresponding cumulative mortality ratios $c \hat{m} r^{(W)}, c \hat{m} r^{(S)}$ and $c \hat{m} r^{(C)}$ may likewise differ from each other, but each may provide a useful long-term summary measure of association with obesity.

The Markov model we studied was meant to be simple, yet realistic enough to give insight into analytical issues. Although the parameters in the model were estimated from NHEFS data, we did not intend that the Markov model be used to analyze obesity data or to describe it fully. The model could be refined in several ways. The states could allow for finer BMI categories and sickness or wellness categories, and one could weaken the Markov assumption. For 
example, it might be that a person who transited from Obese Sick to Non-obese Sick would have a different mortality rate than a person who transited from Non-obese Well to Nonobese Sick; in this case, a second order Markov model might be appropriate. One may also allow some transitions between living states to be reversible, (e.g., the persons can migrate from Non-obese Sick to Obese Sick). Nonetheless, the present model captures several important features. The model allows for individuals to migrate among live states, and, in particular, it allows Obese Sick participants to lose weight and become Non-obese Sick participants. When the only two weight categories are Obese and Non-obese, this transition can reduce the observed adverse association between obesity and mortality over time. Mixtures of Sick and Well participants among the Obese and Non-obese categories at the beginning of follow-up can induce inversions in association of obesity relative risk that can be interpreted as "reverse causation" [13, 18]. Indeed, Flanders and Augestad [5] used a similar model and were able to produce J-shaped curves of age-specific mortality against BMI category. A similar transition probability model was discussed by Diehr et al. [4], who allowed health status to change status either from Well to Sick or from Sick to Well. We restrict Sick status to denote a chronic disease that is not reversible, such as cancer or heart disease.

Excluding participants with a pre-existing disease is equivalent to studying only participants who were Well initially and using the estimator $\hat{r}_{21}^{(W)}$. As discussed previously, this estimator yields unbiased estimates of $r_{21}$ for $F=1$ year, but not for $F=5,10$ or 20 years of follow-up (Figure 3$)$. Over these longer follow-up periods, $\hat{r}_{21}^{(W)}$ reflects the total effect of obesity in an initially well population. Whether one wishes to study the associations with obesity only in Well participants depends on one's beliefs about antecedent causal relationships among obesity and health status. If pre-existing illness caused both lower BMI and increased risk of mortality, one may wish to exclude those with pre-existing illness. If, instead, lower BMI caused the preexisting disease that, in turn, caused increased risk of mortality, then one would not want to exclude those with preexisting disease, which is on the causal pathway from lower BMI to mortality. Another possibility is that pre-existing disease causes lower BMI and this lower BMI increases mortality but not the pre-existing disease; then one would not want to exclude those with pre-existing disease since the pre-existing disease is a surrogate for BMI, the risk factor. Excluding the first five years of mortality experience did not control the bias for estimating in these analyses, in line with results in Allison et al. [1]. However, under our Markov model, the mortality hazard ratios remain constant during follow-up. Singh and Wang [16] allowed the risk of mortality from pre-existing disease to decrease during follow-up and found that excluding the first few years of follow-up reduced bias.

460 B. E. Chen et al.
Caution should be used when applying our results to analyses of the relationship between body size and mortality. Our model captures some aspects of the relationships between body size, health status and mortality rates, but certainly does not describe the full complexity of these relationships, nor allow for heterogeneity in these relationships. Differences in the relationships between body size and mortality have been found for gender [2], race [2] and age groups $[10,11]$, and for health behaviors such as smoking status. In addition, our model has only two BMI groups, Non-obese and Obese, whereas BMI is usually analyzed in five or more categories, such as underweight, normal weight, overweight, moderately obese and very obese. We recommend that researchers examine the effects of a variety of factors, including those considered in this paper, on estimated associations of BMI with mortality. For example, the analysis of Flegal et al. [6] examined the effects of latency, length of follow-up, baseline health status and baseline smoking status. In sensitivity analyses, Flegal et al. limited follow-up and deleted sick individuals and those who had smoked, but found no consistent differences on associations of mortality with BMI from those based on the entire sample with complete followup. In the present paper NHEFS data are used solely to estimate the annual transition probabilities for our Markov model. Our calculations based on this Markov model are not intended to represent an analysis of the relationship between BMI and mortality in the NHEFS Study.

In summary, this paper presents a Markov model that includes BMI, health status and mortality and that allows one to compare different analytic strategies for addressing bias in estimating the relative mortality risk for obese and nonobese individuals. Under this model short term stratumspecific estimates are unbiased for estimating the health stratum-specific instantaneous mortality hazard ratios from obesity, and, updating information on BMI and disease status during long term follow-up reduces bias. For follow-up over 10 disease, excluding the first five years of follow-up, and methods of analysis that ignore health status yield biased estimates of these instantaneous hazard ratios. However, over 10 or 20 year time periods, long-term average mortality hazard ratios or cumulative mortality ratios better reflect the total impact of obesity, including its tendency to accelerate transitions to sickness under this model, than instantaneous mortality hazard ratios. Over these longer time periods, average relative hazard estimates based on initially well participants, $\hat{r}_{21}^{(W)}$, initially sick participants, $\hat{r}_{21}^{(S)}$, and the combined population, $\hat{r}_{21}^{(C)}$, or the corresponding cumulative mortality ratios, $c \hat{m} r^{(W)}, c \hat{m} r^{(S)}$ and $c \hat{m} r^{(C)}$, may each provide valuable descriptions of associations of obesity with mortality.

\section{ACKNOWLEDGEMENTS}

This work was supported in part by the Intramural Research Program of the National Cancer Institute and 
the Natural Sciences and Engineering Research Council of Canada (NSERC). The authors thank the editors and the reviewer for their helpful comments. The authors also thank Dr. David F. Williamson for his helpful comments.

Received 16 September 2010

\section{REFERENCES}

[1] Allison, D. B., Heo, M., Flanders, D. W., Faith, M. S., CarPenter, K. M. and Williamson, D. F. (1999). Simulation study of the effects of excluding early deaths on risk factor-mortality analyses in the presence of confounding due to occult disease: the example of body mass index. Annals of Epidemiology 9 132-142.

[2] Calle, E. E., Thun, M. J., Petrelli, J. M., Rodriguez, C and Heath, JR., C. W. (1999). Body-mass index and mortality in a prospective cohort of u.s. adults. New England Journal of Medicine 341 1097-1105.

[3] Cox, C. S., Mussolino, M. E., Rothwell, S. T., Lane, M. A., Golden, C. D., Madans, J. H. and Feldman, J. J. (Dec 1997). Plan and operation of the NHANES I epidemiologic follow-up study, 1992. Vital Health Stat 135 1-231.

[4] Diehr, P., O’Meara, E. S., Fitzpatrick, A., Newman, A. B., Kuller, L. and Gregory, B. (2007). Weight, mortality, years of healthy life, and active life expectancy in older adults. Journal of American Geriatrics Soc. 55 1-8.

[5] Flanders, W. D. and Augestad, L. B. 2008. Adjusting for reverse causality in the relationship between obesity and mortality. International Journal of Obesity 32 (Suppl 3) 42-46. URL http://dx.doi.org/10.1038/ijo.2008.84.

[6] Flegal, K. M., Graubard, B. I., Williamson, D. F. and Gail, M. H. (Oct 2007). Impact of smoking and preexisting illness on estimates of the fractions of deaths associated with underweight, overweight, and obesity in the US population. American Journal of Epidemiology 166 (8) 975-982. URL http://dx.doi.org/10. 1093/aje/kwm152.

[7] Flegal, K. M., Graubard, B. I., Williamson, D. F. and Gail, M. H. (Apr 2005). Excess deaths associated with underweight, overweight, and obesity. JAMA 293 (15) 1861-1867. URL http://dx.doi.org/10.1001/jama.293.15.1861.

[8] Gompertz, B. (1825). On the nature of the function expressive of the law of human mortality, and on a new mode of determining the value of human contingencies. Philosophical Transactions of the Royal Society of London 27 510-519.

[9] Hernan, M. A. and Taubman, S. L. (2008). Does obesity shorten life? The importance of well-defined interventions to answer causal questions. International Journal of Obesity 32 (Suppl 3) 8-14. URL http://dx.doi.org/10.1038/ijo.2008.82.

[10] Jee, S. H., Sull, J. W., Park, J., Lee, S. Y., Ohrr, H., GualLAR, E. and SAMET, J. M. (2006). Body-mass index and mortality in Korean men and women. New England Journal of Medicine 355 779-787.

[11] Kushner, R. F. (1993). Body weight and mortality. Nutrition Review 51 127-136.
[12] Little, R. J. A. and Rubin, D. B. (2002). Statistical Analysis with Missing Data, 2nd edition. John Wiley, New York. MR1925014

[13] Manson, J. E., Bassuk, S. S., Hu, F. B., Stampfer, M. J., Colditz, G. A. and Willett, W. C. (Mar 2007). Estimating the number of deaths due to obesity: can the divergent findings be reconciled? Journal of Women's Health 16 (2) 168-176. URL http://dx.doi.org/10.1089/jwh.2006.0080.

[14] McGee, D. L. and Diverse Populations Collaboration (2005). Body mass index and mortality: a meta-analysis based on person-level data from twenty-six observational studies. Annals of Epidemiology 15 87-97.

[15] Robins, J. M. (2008). Causal models for estimating the effects of weight gain on mortality. International Journal of Obesity $\mathbf{3 2}$ (Suppl 3) 15-41. URL http://dx.doi.org/10.1038/ijo.2008. 83.

[16] Singh, P. N. and Wang, X. (2001). Simulation study of the effect of an early mortality exclusion on confounding of the exposuremortality relation by preexisting diseases. American Journal of Epidemiology 154 (10) 963-971.

[17] TArone, R. E. (1981). On summary estimators of relative risk. Journal of Chronic Disease 34 463-468.

[18] Willett, W. C., Dietz, W. H. and Colditz, G. A. (Aug 1999). Guidelines for healthy weight. New England Journal of Medicine 341 (6) 427-434.

Bingshu E. Chen

NCIC Clinical Trials Group and

Department of Community Health and Epidemiology

Queens University

Kingston, Ontario

Canada

E-mail address: bechen@ctg.queensu.ca

Barry I. Graubard

Division of Cancer Epidemiology and Genetics

National Cancer Institute

Bethesda, Maryland

USA

Katherine M. Flegal

National Center for Health Statistics

Centers for Disease Control and Prevention

Hyattsville, Maryland

USA

Mitchell H. Gail

Division of Cancer Epidemiology and Genetics

National Cancer Institute

Bethesda, Maryland

USA 